\title{
AVALIAÇÃO DO LITHOTHAMNIUM COMO CORRETIVO DA ACIDEZ DO SOLO E FONTE DE NUTRIENTES PARA O FEIJOEIRO ${ }^{1}$
}

\author{
PAULO CÉSAR DE MELO ${ }^{2}$ \\ ANTONIO EDUARDO FURTINI NETO ${ }^{3}$
}

\begin{abstract}
RESUMO - Com o objetivo de avaliar o efeito do Lithothamnium na correção da acidez de solo e como fonte de nutrientes para o feijoeiro, desenvolveu-se um experimento em condições de casa-de-vegetação. O delineamento experimental utilizado foi o inteiramente casualizado, com quatro repetições, três solos, Neossolo Quartzarênico (NQ), Latossolo Vermelho Amarelo (LV) e Argissolo Vermelho (AV) e nove tratamentos (testemunha dose zero) e quatro doses de Lithothamnium (1/4; $1 / 2 ; 1$ e 2 vezes a dose para $\mathrm{V}$ a $70 \%$ ), e quatro adicionais: calcário dolomítico comercial como referência-padrão (V a $70 \%$ ); Lithothamnium (V a 70\%) sem micronutrientes; Lithothamnium (V a 70\%) com redução de $20 \%$ de NPK e Lithothamnium (V a 70\%) mais Mg. Foram utilizados vasos de três $\mathrm{dm}^{3}$ com quatro plantas de feijoeiro. Dessas quatro, duas foram colhidas no florescimento, nas quais avaliaram-se as concentrações de macro e micronutrientes nas folhas; as outras foram colhidas no final do ciclo, quando avaliou-se, dentre outras características, a produção de matéria seca de grãos. O Litho-
\end{abstract}

thamnium mostrou praticamente o mesmo efeito que o calcário dolomítico comercial na correção da acidez e na saturação por bases utilizando-se a dose para $\mathrm{V}$ a $70 \%$. O Lithothamnium promoveu, nos três solos, a elevação dos teores de cálcio e magnésio, aumento nos valores de $\mathrm{pH}$ e saturação por bases e conseqüente redução na saturação por alumínio, podendo o produto ser utilizado como corretivo e fertilizante. Esses efeitos promoveram melhores condições de nutrição, crescimento e produção do feijoeiro. A dose para se atingir $90 \%$ da produção máxima de grãos de feijoeiro no solo NQ foi de $610 \mathrm{~kg} \mathrm{ha}^{-1}$ e no solo LVd de $1.090 \mathrm{~kg} \mathrm{ha}^{-1}$, abaixo das doses de Lithothamnium para se atingir um $\mathrm{V}$ a 70\%. No NQ, na ausência de Lithothamnium e também no tratamento adicional, Lithothamnium (V a 70\%) sem micronutrientes não houve a produção de grãos. De maneira geral, observou-se que as menores doses de Lithothamnium, nos três solos, foram as que apresentaram melhores resultados nas características avaliadas e que os melhores resultados foram encontrados no LVd.

TERMOS PARA INDEXAÇÃO: Lithothamnium calcareum, Phaseolus vulgaris L., acidez, saturação por bases, fertilizante.

\section{EVALUATION OF LITHOTHAMNIUM AS CORRECTIVE OF SOIL ACIDITY AND SOURCE OF NUTRIENTS FOR BEAN PLANTS}

\begin{abstract}
With the objective of evaluating the efficiency of lithothamnium in the correction of the soil acidity and as source of nutrients for bean plants, an experiment was conducted in vases, in greenhouse conditions. The experimental design was a complete randomized one, with nine treatments: control, four doses of lithothamnium $(1 / 4,1 / 2,1$ and 2 times the dose for $\mathrm{V}$ at $70 \%$ ), commercial dolomite limestone as reference pattern (dose for $\mathrm{V}$ at $70 \%$ ), lithothamnium (V at $70 \%$ ) without micronutrients, lithothamnium (V at
\end{abstract}

$70 \%$ ) with reduction of $20 \%$ of NPK and lithothamnium (V at 70\%) plus Mg. Three soil classes were evaluated, Quartzarenic (NQ), Yellow Red Latossol (LV) and Red Argissol (AV), with four repetitions for each treatment. Four bean plants were cultivated in vases with three $\mathrm{dm}^{3}$. Two of these plants were harvested in the flowering period, with both the macro and micronutrients concentrations being evaluated. The other two plants were harvested at the end of the cycle, when the productions of grain and

1. Parte da Tese de Doutorado do primeiro autor, apresentada à UNIVERSIDADE FEDERAL DE LAVRAS/UFLA, Caixa Postal 37 - 37200-000 - Lavras, MG. Trabalho financiado pela THOTHAM Mineração Marítima Ltda.

2. Engenheiro Agrônomo - Pesquisador, Professor no Instituto de Ciências Agrárias da Universidade Federal de Uberlândia - ICIAG/UFU. pcmelo@ufu.br

3. Professor Doutor do Departamento de Ciência do Solo, UFLA. afurtini@uflabr 
vegetable dry matter (aerial parts, root, grain and total) were evaluated. Lithothamnium showed practically the same effect of the commercial dolomite limestone in the correction of the acidity and in the saturation for bases being used the dose to reach a V at $70 \%$. Lithothamnium promoted in the three soils, the elevation of calcium and magnesium levels, increasing the $\mathrm{pH}$ values and saturation for bases, and consequently, reduction in the saturation for aluminum, accrediting the product to be used as corrective and fertilizer. Those effects promoted better nutrition conditions, growth and production of the bean plant.
The doses to reach $90 \%$ of the maximum production of grains in the NQ and LV soils were 610 and $1.090 \mathrm{~kg}$ $\mathrm{ha}^{-1}$, respectively, these doses being lower than those required to reach a $\mathrm{V}$ at $70 \%$. In $\mathrm{NQ}$ soil, without lithothamnium, and also in the treatment with lithothamnium (dose for $\mathrm{V}$ at $70 \%$ ) without micronutrients, plants did not produce grains. In a general way, it was observed that the smallest doses of lithothamnium, in the three soils, were the ones that presented better results in the appraised characteristics and that the best results were found in LV.

INDEX TERMS: Lithothamnium calcareum, Phaseolus vulgaris L., soil acidity, saturation for bases, fertilizer.

\section{INTRODUÇÃO}

Há muito tempo se tem utilizado o Lithothamnium, material corretivo derivado de algas marinhas calcárias, nas costas francesa, inglesa e irlandesa para correção de solos ácidos e/ou deficientes em cálcio. Nessas regiões, o produto é conhecido pelo nome de Calcified Seaweed ou "maërl", sendo composto de esqueleto remanescente de Phytamolithium calcareum e Lithothamnium corraloides. Estudos antigos, datados do ano 1853, na Europa, indicam que seu uso parece ter tido uma primeira menção no ano 1186 (Le Bleu, 1983).

No Brasil, os depósitos de algas calcificadas do grupo das Melobesiae são encontrados desde a Região Amazônica até o sul do Rio de Janeiro, numa extensão de cerca de $4.000 \mathrm{~km}$, com reservas ainda não conhecidas. Esses fundos de Melobesiae, livres da plataforma continental, localizam-se próximo ao litoral, e com sua relativa facilidade de exploração e processamento, podem-se constituir em alternativa de produto para fins agrícolas (Kempf, 1974).

O produto é retirado do fundo do mar, do sedimento marinho, na plataforma continental do Espirito Santo e estocado no pátio da fabrica por um período variável. Após a primeira trituração, é seco ao ar quente e micropulverizado a frio. Devido à porosidade do corpo da alga, o produto apresenta uma atividade muito intensa no solo, atividade essa devida principalmente à elevada superfície específica do material (Lopez-Benito, 1963).

Miranda (1985) estudou a utilização de um calcário magnesiano comercial e dois calcários marinhos (Lithothamne C e Lithothamne 400), de procedência francesa, como corretivos da acidez do solo para a cultura do milho-grão, em dois Latossolos. Concluiu que os calcários marinhos são viáveis como corretivos da acidez do solo quando aplicados em quantidades semelhantes às do calcário comercial. No entanto, seu uso não dispensaria a aplicação de micronutrientes para solos deficientes nesses elementos. $\mathrm{O}$ mesmo autor sugere que seria importante que os calcários marinhos fossem testados em campo, para avaliar o seu efeito residual e, dependendo dos custos de sua extração e preparo, possam constituir uma alternativa de corretivo de acidez do solo nas regiões agrícolas próximas do litoral.

Rosolem (1987) discute os efeitos da calagem no feijoeiro, apontando os efeitos positivos dessa prática na nutrição nitrogenada (via aumento da disponibilidade de Mo, afetando a fixação simbiótica e a assimilação do $\mathrm{N}$ mineral absorvido ou em função do maior desenvolvimento radicular) na economia de fertilizante fosfatado, na correção dos níveis tóxicos de $\mathrm{Al}$ e $\mathrm{Mn}$, na prevenção da deficiência de micronutrientes e na maior tolerância a condições hídricas adversas.

Com o presente trabalho, objetivou-se avaliar o efeito do Lithothamnium sobre os atributos químicos relacionados à acidez do solo e como fonte de nutrientes para o feijoeiro (Phaseolus vulgaris L.) cultivado em três solos distintos.

\section{MATERIAL E MÉTODOS}

O experimento foi conduzido em condições de casa-de-vegetação do DCS-UFLA. O Lithothamnium foi caracterizado e analisado quimicamente (Quadro 1).

Foram coletadas amostras $(0$ a $20 \mathrm{~cm})$ de três solos abrangendo três classes: Neossolo Quartzarênico (NQ), Latossolo Vermelho Amarelo distrófico, textura média (LVd) e Argissolo Vermelho distrófico, textura muito argilosa (AVd). As amostras foram secas ao ar, peneiradas em malha de $5 \mathrm{~mm}$ de abertura, e caracterizadas química e fisicamente (Quadro 2). 
QUADRO 1 - Caracterização química do Lithothamnium.

\begin{tabular}{|c|c|c|c|c|c|c|}
\hline $\mathrm{CaO}$ & $\mathbf{C a}$ & MgO & Mg & PN & Reatividade & PRNT \\
\hline \multicolumn{7}{|c|}{ - } \\
\hline 462,7 & 330,5 & 42,3 & 25,4 & 93,31 & 99,26 & 92,62 \\
\hline
\end{tabular}

${ }^{1}$ Análises realizadas no Laboratório de Análise de Corretivos do DQI/UFLA, conforme metodologia descrita em Brasil (1983).

QUADRO 2 - Principais características químicas e físicas dos solos utilizados, antes da aplicação dos tratamentos ${ }^{1}$.

\begin{tabular}{|c|c|c|c|}
\hline \multirow{2}{*}{ Características } & \multicolumn{3}{|c|}{ Solo } \\
\hline & NQ & LVd & Avd \\
\hline pH em água $(1: 2,5)$ & 5,1 & 4,8 & 4,7 \\
\hline $\mathrm{P} \quad\left(\mathrm{mg} \mathrm{dm}^{-3}\right)$ & 2,0 & 1,0 & 2,0 \\
\hline $\mathrm{K} \quad\left(\mathrm{mg} \mathrm{dm}^{-3}\right)$ & 16,0 & 16,0 & 31,0 \\
\hline $\mathrm{Ca} \quad\left(\mathrm{mmol}_{\mathrm{c}} \mathrm{dm}^{-3}\right)$ & 4,0 & 6,0 & 10,0 \\
\hline $\operatorname{Mg}\left(\mathrm{mmol}_{\mathrm{c}} \mathrm{dm}^{-3}\right)$ & 1,0 & 2,0 & 4,0 \\
\hline $\mathrm{Al} \quad\left(\mathrm{mmol}_{\mathrm{c}} \mathrm{dm}^{-3}\right)$ & 8,0 & 10,0 & 13,0 \\
\hline $\mathrm{H}+\mathrm{Al}\left(\mathrm{mmol}_{\mathrm{c}} \mathrm{dm}^{-3}\right)$ & 29,0 & 50,0 & 63,0 \\
\hline $\mathrm{S}\left(\mathrm{mmol}_{\mathrm{c}} \mathrm{dm}^{-3}\right)$ & 5,0 & 8,0 & 15,0 \\
\hline CTC a pH 7,0-T $\left(\mathrm{mmol}_{\mathrm{c}} \mathrm{dm}^{-3}\right)$ & 34,0 & 58,0 & 78,0 \\
\hline Saturação por Al (\%) & 59,7 & 54,3 & 46,8 \\
\hline Saturação por bases $(\%)$ & 15,7 & 14,4 & 19,0 \\
\hline $\mathrm{S}-$ Sulfato $\left(\mathrm{mg} \mathrm{dm}^{-3}\right)$ & 2,3 & 0,8 & 4,7 \\
\hline B $\quad\left(\mathrm{mg} \mathrm{dm}^{-3}\right)$ & 0,1 & 0,1 & 0,4 \\
\hline $\mathrm{Zn} \quad\left(\mathrm{mg} \mathrm{dm}^{-3}\right)$ & 0,6 & 0,5 & 0,1 \\
\hline $\mathrm{Cu} \quad\left(\mathrm{mg} \mathrm{dm}^{-3}\right)$ & 0,4 & 1,4 & 0,4 \\
\hline $\operatorname{Mn}\left(\mathrm{mg} \mathrm{dm}^{-3}\right)$ & 1,6 & 0,4 & 1,9 \\
\hline $\mathrm{Fe} \quad\left(\mathrm{mg} \mathrm{dm}^{-3}\right)$ & 90,1 & 101,4 & 103,4 \\
\hline Matéria orgânica $\left(\mathrm{g} \mathrm{kg}^{-1}\right)$ & 13,0 & 21,0 & 31,0 \\
\hline Argila $\left(\mathrm{g} \mathrm{kg}^{-1}\right)$ & 20 & 330 & 830 \\
\hline Areia $\left(\mathrm{g} \mathrm{kg}^{-1}\right)$ & 950 & 520 & 70 \\
\hline Silte $\left(\mathrm{g} \mathrm{kg}^{-1}\right)$ & 30 & 150 & 100 \\
\hline Classe Textural & Arenosa & Média & Muito argilosa \\
\hline
\end{tabular}

${ }^{1}$ - Análises realizadas no DCS/UFLA. As concentrações de P, Ca, Mg, Al e $\mathrm{K}$ foram determinadas com modificações propostas pela EMBRAPA. Os micronutrientes $\mathrm{Cu}$, Fe, Mn e $\mathrm{Zn}$ foram extraídos pelo DTPA e quantificados por espectrofotometria de absorção atômica. $O$ boro foi extraído através da metodologia da água quente (Berger \& Truog, 1939). 
Utilizou-se delineamento inteiramente casualizado com quatro repetições e três solos (NQ, LVd e AVd) e 9 tratamentos resumidos no Quadro 3.

O Lithothamnium e o calcário foram aplicados em forma sólida e os macro e micronutrientes foram aplicados na forma de solução, fazendo-se cuidadosa homogeneização do material. Dessa forma, os tratamentos foram aplicados, adubando-se no plantio com macro e micronutrientes $\left(\mathrm{mg} \mathrm{dm}^{-3}\right): 100$ de $\mathrm{N} ; 150$ de K; 50 de $\mathrm{S} ; 0,8$ de B; 1,3 de $\mathrm{Cu}$; 4,0 de Fe; 3,6 de Mn e 5,0 de Zn e, no caso do fósforo, forneceram-se 150, 250 e $350 \mathrm{mg}$ de $\mathrm{P} \mathrm{dm}^{-3}$ para os solos NQ, LVd e AVd, respectivamente. Como fontes, utilizaram-se $\mathrm{NH}_{4} \mathrm{H}_{2} \mathrm{PO}_{4} ; \mathrm{K}_{2} \mathrm{SO}_{4} ; \mathrm{KCl}$; $\mathrm{NH}_{4} \mathrm{NO}_{3} ; \mathrm{H}_{3} \mathrm{PO}_{4} ; \mathrm{H}_{3} \mathrm{BO}_{3} ; \mathrm{CuCl}_{2} \cdot 2 \mathrm{H}_{2} \mathrm{O} ; \mathrm{FeCl}_{3} \cdot 6 \mathrm{H}_{2} \mathrm{O}$; $\mathrm{MnSO}_{4} \mathrm{H}_{2} \mathrm{O}$ e $\mathrm{ZnCl}_{2}$, na forma de sais p.a. Os solos com os tratamentos foram incubados por 30 dias, com umidade $70 \%$ do volume total de poros, VTP (Freire et al., 1980). Após incubação, fez-se amostragem dos solos por vaso para as determinações analíticas pré-plantio. Semearam-se 9 sementes de feijão carioca, cultivar "Milênio", hábito de crescimento indeterminado, tipo 3 (Departamento de Biologia/UFLA). A umidade do solo foi mantida em torno de $70 \%$ do VTP, por meio de pesagens periódicas com adição de água deionizada. Após a emergência, estádio R1, desbastaram-se e conduziramse quatro plantas por vaso $\left(3,0 \mathrm{dm}^{3}\right.$ de solo $)$. Os vasos foram submetidos a um rodízio semanal em sua posição na casa-de-vegetação. Foram realizadas adubações em cobertura totalizando $540 \mathrm{mg} \mathrm{dm}^{-3}$ de $\mathrm{N}$ e $240 \mathrm{mg} \mathrm{dm}^{-3}$ de $\mathrm{K}$ na forma de $\mathrm{NH}_{4} \mathrm{NO}_{3}$ e $\mathrm{KNO}_{3}$, sais p.a., com base em Feitosa (1980). No período do florescimento (R6), foram coletadas duas plantas, cortando-as rente ao solo, para análise dos teores de macro e micronutrientes nas folhas, segundo Malavolta et al. (1997).

A colheita das vagens na fase de maturação foi realizada aos 75 dias após a emergência. Após a colheita, todo material vegetal foi separado em parte aérea (caule + ramos + folhas + vagens), raiz e grãos, sendo lavado, seco e acondicionado em sacos de papel. Posteriormente, foi seco em estufa de circulação de ar forçado na temperatura de $60^{\circ}$ a $65^{\circ} \mathrm{C}$, até atingir massa constante.

Os dados obtidos foram submetidos à analise de variância e como ocorreram diferenças significativas pelo teste $\mathrm{F}$, teste de médias (Tukey), teste $\mathrm{t}$ para contraste entre duas médias e, nas doses crescentes, estudos de regressão polinomial foram efetuados com o objetivo de analisar o efeito isolado das doses de Lithothamnium em relação à testemunha, de acordo com Banzato \& Kronka (1989). Equações de regressão foram ajustadas às médias de massa de matéria seca de grãos em função das doses de Lithothamnium aplicadas. Por meio dessas equações de regressão, obtiveram-se as estimativas das doses de Lithothamnium para produção de $90 \%$ da máxima.

\section{RESULTADOS E DISCUSSÃO}

Os resultados da análise química dos solos são apresentados no Quadro 4.

QUADRO 3 - Tratamentos utilizados no experimento com o feijoeiro.

\begin{tabular}{|c|c|c|c|c|c|c|c|}
\hline & \multirow[b]{2}{*}{ Tratamento } & \multicolumn{3}{|c|}{ Solo } & \multirow{2}{*}{$\begin{array}{c}\text { Adubação } \\
\text { básica } \\
\text { NPK } \\
\end{array}$} & \multirow{2}{*}{$\begin{array}{c}\text { Adubação } \\
\text { micro- } \\
\text { nutrientes }\end{array}$} & \multirow{2}{*}{$\begin{array}{l}\text { Adição } \\
\text { de Mg }\end{array}$} \\
\hline & & NQ & $\mathbf{L V}$ & AV & & & \\
\hline \multicolumn{8}{|c|}{ Quantidade do Produto $\left(\right.$ g vaso $\left.^{-1}\right)$} \\
\hline $\mathrm{T} 1$ & Litho Dose 0 & 0 & 0 & 0 & + & + & - \\
\hline $\mathrm{T} 2$ & Litho Dose 1 & $1,3(0,88)^{2}$ & $2,3(1,52)$ & $2,9(1,96)$ & + & + & - \\
\hline $\mathrm{T} 3$ & Litho Dose 2 & $2,6(1,72)$ & $4,6(3,08)$ & $5,8(3,92)$ & + & + & - \\
\hline $\mathrm{T} 4$ & Litho Dose 3 & $5,2(3,48)$ & $9,2(6,12)$ & $11,7(7,80)$ & + & + & - \\
\hline T5 & Litho Dose 4 & $10,4(6,96)$ & $18,4(12,24)$ & $23,4(15,60)$ & + & + & - \\
\hline T6 & Calcário dolomítico ${ }^{1}$ & $4,6(3,04)$ & $8,0(5,32)$ & $10,4(6,92)$ & + & + & - \\
\hline $\mathrm{T} 7$ & Lithodose 3 & $5,2(3,48)$ & $9,2(6,12)$ & $11,7(7,80)$ & + & - & - \\
\hline $\mathrm{T} 8$ & Lithodose 3 & $5,2(3,48)$ & $9,2(6,12)$ & $11,7(7,80)$ & $-20 \%$ & + & - \\
\hline T9 & Lithodose 3 & $5,2(3,48)$ & $9,2(6,12)$ & $11,7(7,80)$ & + & + & + \\
\hline
\end{tabular}

${ }^{1}$ Dose de calcário dolomítico como referência para comparações.

2 Toneladas por ha. 
QUADRO 4 - Caracterização química das amostras dos solos, após incubação com os tratamentos.

\begin{tabular}{|c|c|c|c|c|c|c|c|c|c|c|}
\hline \multirow{2}{*}{ Solo } & \multirow{2}{*}{ Características } & \multicolumn{9}{|c|}{ Tratamentos } \\
\hline & & $\mathbf{T}_{1}$ & $\mathbf{T}_{2}$ & $\mathbf{T}_{\mathbf{3}}$ & $\mathbf{T}_{4}$ & $\mathbf{T}_{5}$ & $\mathbf{T}_{6}$ & $\mathbf{T}_{7}$ & $\mathbf{T}_{8}$ & $\mathbf{T}_{9}$ \\
\hline \multirow[t]{19}{*}{ NQ } & pH em água $(1: 2,5)$ & 5,7 & 6,0 & 6,0 & 6,1 & 6,7 & 6,3 & 6,0 & 6,0 & 6,3 \\
\hline & P Mehlich $1 \quad\left(\mathrm{mg} \mathrm{dm}^{-3}\right)$ & 88 & 95 & 89 & 88 & 89 & 88 & 88 & 70 & 80 \\
\hline & K Mehlich $1\left(\mathrm{mg} \mathrm{dm}^{-3}\right)$ & 132 & 159 & 135 & 138 & 137 & 124 & 134 & 114 & 127 \\
\hline & $\mathrm{Ca} \quad\left(\mathrm{mmol}_{\mathrm{c}} \mathrm{dm}^{-3}\right)$ & 3 & 7 & 10 & 18 & 31 & 11 & 18 & 19 & 17 \\
\hline & $\operatorname{Mg}\left(\mathbf{m m o l}_{\mathbf{c}} \mathbf{d m}^{-3}\right)$ & 1 & 2 & 3 & 5 & 4 & 9 & 7 & 5 & 10 \\
\hline & $\mathrm{Ca}: \mathrm{Mg}$ & 3 & 3,5 & 3,3 & 3,6 & 7,7 & 1,2 & 2,6 & 3,8 & 1,7 \\
\hline & $\mathrm{Al}\left(\mathbf{m m o l}_{\mathrm{c}} \mathbf{d m}^{-3}\right)$ & 3 & 1 & $\mathbf{0}$ & $\mathbf{0}$ & $\mathbf{0}$ & $\mathbf{0}$ & $\mathbf{0}$ & $\mathbf{0}$ & $\mathbf{0}$ \\
\hline & $\mathrm{H}+\mathrm{Al}\left(\mathrm{mmol}_{\mathrm{c}} \mathrm{dm}^{-3}\right)$ & 25 & 21 & 18 & 15 & 11 & 16 & 16 & 15 & 13 \\
\hline & CTC efetiva $-t \quad\left(\mathbf{m m o l}_{\mathbf{c}} \mathbf{d m}^{-3}\right)$ & 10 & 14 & 17 & 27 & 39 & 24 & 29 & 28 & 31 \\
\hline & CTC a pH 7,0-T $\left(\mathrm{mmol}_{\mathrm{c}} \mathrm{dm}^{-3}\right)$ & 33 & 30 & 30 & 41 & 49 & 40 & 44 & 43 & 44 \\
\hline & Saturação por Al - (\%) & 27 & 6 & $\mathbf{0}$ & $\mathbf{0}$ & $\mathbf{0}$ & $\mathbf{0}$ & $\mathbf{0}$ & $\mathbf{0}$ & $\mathbf{0}$ \\
\hline & Saturação por bases - (\%) & 23 & 39 & 48 & 65 & 78 & 59 & 65 & 66 & 70 \\
\hline & Matéria orgânica $\left(\mathrm{g} \mathrm{dm}^{-3}\right)$ & 14 & 14 & 14 & 13 & 14 & 13 & 13 & 13 & 14 \\
\hline & $\mathrm{S}-\mathrm{SO}_{4}\left(\mathrm{mg} \mathrm{dm}^{-3}\right)$ & 49 & 65 & 62 & 79 & 80 & 50 & 53 & 53 & 61 \\
\hline & $B \quad\left(\mathrm{mg} \mathrm{dm}^{-3}\right)$ & 0,5 & 0,7 & 0,8 & 1 & 1 & 0,8 & 0,5 & 1 & 0,9 \\
\hline & $\mathrm{Cu}\left(\mathrm{mg} \mathrm{dm}^{-3}\right)$ & 3,1 & 2,9 & 1,6 & 1,3 & 1,6 & 0,5 & 0,9 & 0,7 & 0,5 \\
\hline & $\mathrm{Fe} \quad\left(\mathbf{m g ~ d m} \mathbf{m}^{-3}\right)$ & 94 & 65 & 53 & 50 & 27 & 52 & 43 & 50 & $\mathbf{5 0}$ \\
\hline & $\operatorname{Mn}\left(\mathrm{mg} \mathrm{dm}^{-3}\right)$ & 6,5 & 4,5 & 3,6 & 4,3 & 3,1 & 4,1 & 2,7 & 4,3 & 2,8 \\
\hline & $\mathrm{Zn} \quad\left(\mathbf{m g ~ d m} \mathbf{m}^{-3}\right)$ & 2,8 & 4,8 & 1,6 & 1 & 0,8 & 1,2 & 1 & 0,7 & 0,9 \\
\hline \multirow[t]{19}{*}{ LVd } & pH em água $(1: 2,5)$ & 5,2 & 5,6 & 5,9 & 6,2 & 6,9 & 6,2 & 6,1 & 6,1 & 6,1 \\
\hline & P Mehlich $1 \quad\left(\mathrm{mg} \mathrm{dm}^{-3}\right)$ & 54 & 46 & 52 & 48 & 51 & 57 & 56 & 34 & 29 \\
\hline & K Mehlich $1 \quad\left(\mathrm{mg} \mathrm{dm}^{-3}\right)$ & 172 & 157 & 167 & 161 & 162 & 202 & 192 & 164 & 190 \\
\hline & $\mathrm{Ca} \quad\left(\mathrm{mmol}_{\mathbf{c}} \mathbf{d m}^{-3}\right)$ & 4 & 11 & 20 & 36 & 60 & 32 & 45 & 44 & 43 \\
\hline & $\operatorname{Mg}\left(\mathrm{mmol}_{\mathrm{c}} \mathrm{dm}^{-3}\right)$ & 2 & 5 & 8 & 6 & 10 & 25 & 9 & 8 & 24 \\
\hline & Ca:Mg & 2 & 2,2 & 2,5 & 6 & 6 & 1,3 & 5 & 5,5 & 1,8 \\
\hline & $\mathrm{Al}\left(\mathrm{mmol}_{\mathrm{c}} \mathrm{dm}^{-3}\right)$ & 4 & 1 & 1 & 0 & 0 & 0 & 0 & 0 & 0 \\
\hline & $\mathbf{H}+\mathbf{A l}\left(\mathbf{m m o l}_{\mathbf{c}} \mathbf{d m}^{-3}\right)$ & 58 & 37 & 30 & 22 & 14 & 25 & 25 & 24 & 22 \\
\hline & CTC efetiva $-\mathrm{t} \quad\left(\mathrm{mmol}_{\mathrm{c}} \mathrm{dm}^{-3}\right)$ & 15 & 21 & 32 & 46 & 74 & 62 & 59 & 57 & 72 \\
\hline & CTC a pH 7,0 - T $\left(\mathrm{mmol}_{\mathrm{c}} \mathrm{dm}^{-3}\right)$ & 68 & 57 & 62 & 68 & 88 & 88 & 83 & 80 & 93 \\
\hline & Saturação por Al - (\%) & 29 & 5 & 2 & 0 & 0 & 0 & 0 & 0 & 0 \\
\hline & Saturação por bases - (\%) & 15 & 35 & 52 & 67 & 84 & 72 & 71 & 70 & 77 \\
\hline & Matéria orgânica $\left(\mathrm{g} \mathrm{dm}^{-3}\right)$ & 30 & 24 & 26 & 26 & 27 & 32 & 33 & 32 & 32 \\
\hline & $\mathrm{S}-\mathrm{SO}_{4}\left(\mathrm{mg} \mathrm{dm}^{-3}\right)$ & 73 & 70 & 92 & 105 & 147 & 147 & 138 & 88 & 75 \\
\hline & B $\left(\mathrm{mg} \mathrm{dm}^{-3}\right)$ & 0,6 & 0,8 & 0,7 & 0,7 & 0,6 & 0,5 & 0,5 & 0,7 & 0,8 \\
\hline & $\mathrm{Cu}\left(\mathrm{mg} \mathrm{dm}^{-3}\right)$ & 1,8 & 1,2 & 1,6 & 0,9 & 0,5 & 0,9 & 0,3 & 0,6 & 0,5 \\
\hline & $\mathrm{Fe} \quad\left(\mathrm{mg} \mathrm{dm}^{-3}\right)$ & 125 & 94 & 84 & 45 & 24 & 51 & 49 & 55 & 39 \\
\hline & $\operatorname{Mn}\left(\mathbf{m g ~ d m}^{-3}\right)$ & 5,9 & 3,9 & 3,2 & 4,2 & 3,7 & 4,9 & $\mathbf{3 , 3}$ & 4,7 & 4 \\
\hline & $\mathrm{Zn} \quad\left(\mathrm{mg} \mathrm{dm}^{-3}\right)$ & 2,4 & 1,8 & 1,3 & 1,2 & 1 & 2,1 & 0,1 & 1,3 & 1,6 \\
\hline
\end{tabular}

Continua..

Ciênc. agrotec., Lavras. V.27, n.3, p.508-519, maio/jun., 2003 
QUADRO 4 - Continuação...

\begin{tabular}{|c|c|c|c|c|c|c|c|c|c|c|}
\hline \multirow[t]{2}{*}{ AVd } & pH em água $(1: 2,5)$ & 4,7 & 5 & 5,3 & 5,7 & 6,9 & 5,9 & $\mathbf{5 , 9}$ & 5,8 & 5,9 \\
\hline & P Mehlich $1 \quad\left(\mathrm{mg} \mathrm{dm}^{-3}\right)$ & 112 & 121 & 121 & 116 & 118 & 113 & 119 & 87 & 101 \\
\hline & K Mehlich $1\left(\mathrm{mg} \mathrm{dm}^{-3}\right)$ & 173 & 174 & 178 & 189 & 192 & 191 & 197 & 165 & 182 \\
\hline & $\mathrm{Ca} \quad\left(\mathrm{mmol}_{\mathrm{c}} \mathrm{dm}^{-3}\right)$ & 12 & 24 & 37 & 60 & 88 & 42 & 59 & 55 & 56 \\
\hline & $\operatorname{Mg}\left(\mathbf{m m o l}_{\mathrm{c}} \mathrm{dm}^{-3}\right)$ & 2 & 8 & 6 & 10 & 10 & 30 & 11 & 11 & 24 \\
\hline & $\mathrm{Ca}: \mathrm{Mg}$ & 6 & 3 & 6,2 & 6 & 8,8 & 1,4 & 5,4 & 5 & 2,3 \\
\hline & $\mathrm{Al}\left(\mathbf{m m o l}_{\mathrm{c}} \mathbf{d m}^{-3}\right)$ & 4 & 1 & 1 & $\mathbf{0}$ & $\mathbf{0}$ & $\mathbf{0}$ & $\mathbf{0}$ & $\mathbf{0}$ & $\mathbf{0}$ \\
\hline & $\mathrm{H}+\mathrm{Al}\left(\mathrm{mmol}_{\mathrm{c}} \mathrm{dm}^{-3}\right)$ & 58 & 37 & 30 & 22 & 14 & 25 & 25 & 24 & 22 \\
\hline & CTC efetiva - $t \quad\left(\mathbf{m m o l}_{\mathbf{c}} \mathbf{d m}^{-3}\right)$ & 15 & 21 & 32 & 46 & 74 & 62 & 59 & 57 & 72 \\
\hline & CTC a pH 7,0-T $\left(\mathrm{mmol}_{\mathrm{c}} \mathrm{dm}^{-3}\right)$ & 109 & 98 & 96 & 105 & 116 & 107 & 103 & 98 & 112 \\
\hline & Saturação por Al - (\%) & 36 & 8 & 1 & $\mathbf{0}$ & $\mathbf{0}$ & $\mathbf{0}$ & $\mathbf{0}$ & $\mathbf{0}$ & $\mathbf{0}$ \\
\hline & Saturação por bases - (\%) & 17 & 36 & 49 & 72 & 89 & 71 & 73 & 71 & 76 \\
\hline & Matéria orgânica $\left(\mathrm{g} \mathrm{dm}^{-3}\right)$ & 23 & 32 & 33 & 31 & 30 & 32 & 32 & 33 & 32 \\
\hline & $\mathrm{S}-\mathrm{SO}_{4}\left(\mathrm{mg} \mathrm{dm}^{-3}\right)$ & 76 & 86 & 97 & 112 & 148 & 110 & 128 & 106 & 162 \\
\hline & $B \quad\left(\mathrm{mg} \mathrm{dm}^{-3}\right)$ & 0,5 & $\mathbf{0 , 5}$ & 0,6 & 0,7 & 0,6 & 0,6 & $\mathbf{0 , 3}$ & 0,5 & 0,8 \\
\hline & $\mathrm{Cu}\left(\mathrm{mg} \mathrm{dm}^{-3}\right)$ & 0,6 & 1,4 & 0,6 & 1,7 & 0,4 & 0,5 & 0,3 & 0,5 & 1,1 \\
\hline & $\mathrm{Fe}\left(\mathrm{mg} \mathrm{dm}^{-3}\right)$ & 80 & 80 & 65 & 32 & 15 & 41 & 35 & 34 & 29 \\
\hline & $\operatorname{Mn}\left(\mathrm{mg} \mathrm{dm}^{-3}\right)$ & 6,5 & 5,8 & 4,2 & 2,4 & 3,2 & 2,2 & 1,6 & 1,5 & 2,2 \\
\hline & $\mathrm{Zn}\left(\mathrm{mg} \mathrm{dm}^{-3}\right)$ & 2,7 & 2 & 1,1 & 0,6 & 0,5 & 1,3 & 0,2 & $\mathbf{0 , 7}$ & 0,9 \\
\hline
\end{tabular}

${ }^{1}$ - Análises realizadas no Laboratório de Fertilidade do Solo, DCS/UFLA. ${ }^{2}$ T1 ao T5 = Doses de Lithothamnium, $\mathrm{T6}^{*}=$ dose de calcário dolomítico $\mathrm{V}$ a 70\%, $\mathrm{T} 7$ = Lithodose 3 sem micro, $\mathrm{T8}=$ Lithodose 3 menos $20 \%$ NPK e T9 $=$ Lithodose $3+$ Mg.

Os solos apresentaram a mesma tendência de elevação de $\mathrm{pH}$, neutralização do $\mathrm{Al}^{3+}$ trocável e incremento de $\mathrm{Ca}^{2+}$ e $\mathrm{Mg}^{2+}$. Entretanto, para o calcário dolomítico, na dose para $\mathrm{V}$ a $70 \%$, o $\mathrm{pH}$ atingiu 6,3 , valor próximo dos outros tratamentos no solo NQ, à exceção do tratamento $\mathrm{T} 5$.

Para a relação Ca:Mg apresentada no Quadro 4, para os três solos, houve uma tendência de aumento com as respectivas doses crescentes do Lithothamnium, tratamentos T2 a T5. Nos tratamentos com Lithothamnium, os valores dessa relação variaram de 1,3 a 10,6 no solo NQ, tratamentos T3 + Mg e T5. Vale ressaltar que, para o calcário dolomítico (V a $70 \%$ ), a relação variou de 1,$5 ; 1,2$ e 1,9 nos solos $\mathrm{NQ}, \mathrm{LVd}$ e AVd, respectivamente, sendo indiferentes para a cultura do feijoeiro (Bornman et al., 1998).

$\mathrm{Na}$ redução da saturação por alumínio $(\mathrm{m} \%)$ também ocorreu a mesma tendência nos três solos, com valores menores em relação à testemunha (Quadro 4).

\section{Produção de grãos (MSGRÃO)}

Os tratamentos influenciaram significativamente $(\mathrm{P}<0,05)$ a produção de grãos nos solos NQ e LVd (Quadro 5).

Em todos os solos, a produção de matéria seca de grãos foi menor (ou mesmo ausente) no tratamentotestemunha, comprovando a grande sensibilidade do feijoeiro à acidez do solo. Os dados de MSGRÃO do feijoeiro no solo AVd não se ajustaram a nenhum modelo de regressão.

Na Figura 1 fica demonstrado que, nos solos NQ e $L V d$, o feijoeiro respondeu significativamente à aplicação do Lithothamnium, com elevados incrementos na produção de MSGRÃO, nas doses iniciais, seguido de um patamar de estabilização, após atingir o máximo.

A produção de matéria seca de grãos em função das doses de Lithothamnium seguiu o modelo de resposta base raiz quadrada nos solos (Figura 1). Ela foi máxima nas doses de 5,06 e 17,04 $\mathrm{g} \mathrm{vaso}^{-1}$, para os solos NQ e LVd, respectivamente (Quadro 6). Para o solo NQ, a quantidade de Lithothamnium para $90 \%$ da pro- 
dução máxima situou-se bastante próxima da primeira dose do produto (Quadro 3), a qual proporcionou $\mathrm{pH}$ de 6,0 e valor de saturação por bases (Quadro 4) abaixo do adequado para a cultura do feijoeiro, de acordo com Raij et al. (1996). Entretanto, para o solo LVd, a quantidade de Lithothamnium para $90 \%$ da produção máxima situou-se acima da primeira dose, mais próxima da segunda dose, a qual proporcionou valor de saturação por bases de 52\% (Quadro 4). Essa dose, teste F ( $\mathrm{P}<0,05 \%)$ não alterou a produção de matéria seca de grão (Figura 1). Os modelos indicam aumento na produção de matéria seca de grãos com aplicação das doses crescentes do
Lithothamnium até atingir um ponto máximo, o qual não varia a produção, mesmo com as doses mais altas.

As estimativas de produções de matéria seca de grãos (90\% da produção máxima) corresponderam às doses de 612 e $1096 \mathrm{~kg} \mathrm{ha}^{-1}$ do produto, representando uma diminuição de $69,8 \%$ e $83,9 \%$ em relação à dose para máxima produção, nos solos NQ e LVd (Quadro 6). Esta redução em mais de um terço na quantidade de Lithothamnium pode significar expressiva redução de custos nas práticas corretivas do solo.

A taxa de beneficio no solo NQ apresentou-se menor que o solo LVd por grama do Lithothamnium aplicado (Quadro 6), mas foi altamente expressiva.

QUADRO 5 - Resumo da análise de variância para a característica MSGRÃO.

\begin{tabular}{|c|c|c|c|c|}
\hline \multirow{3}{*}{ F.V. } & \multirow{3}{*}{ GL } & \multicolumn{3}{|c|}{ Solo } \\
\hline & & NQ & LVd & AVd \\
\hline & & \multicolumn{3}{|c|}{...QM.. } \\
\hline Tratamento & 8 & $54,4985 * *$ & $66,52805^{*}$ & $140,9954 * *$ \\
\hline Erro & 27 & 5,7748 & 4,1164 & 18,3212 \\
\hline C.V. $(\%)$ & - & 19,2 & 19,2 & 22,9 \\
\hline
\end{tabular}

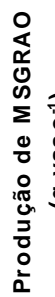

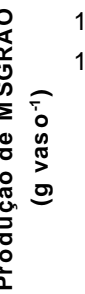

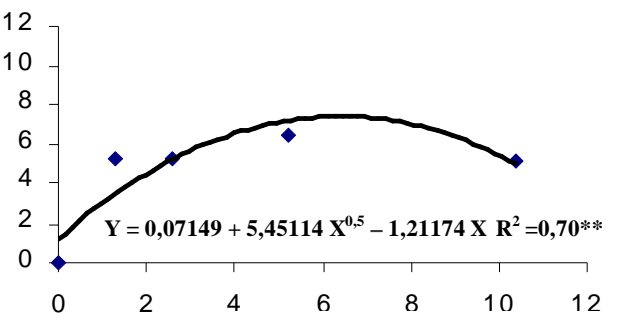

Doses de Lithothamnium $\left(\mathrm{g}_{\text {vaso }}{ }^{-1}\right)$

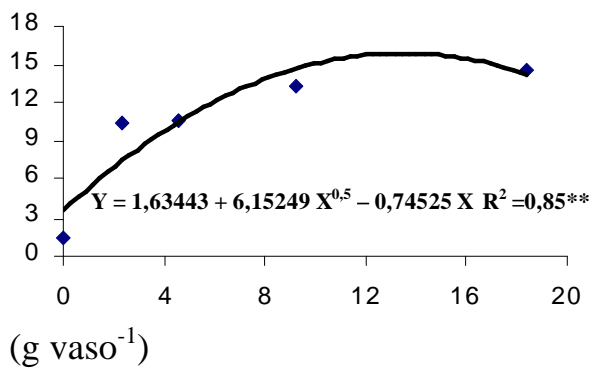

FIGURA 1 - Produção de matéria seca de grãos do feijoeiro em função das doses de Lithothamnium nos solos NQ, $\operatorname{LVd}(* *$ significativo a $1 \%$, teste $\mathrm{t})$.

QUADRO 6 - Massa estimada da produção de matéria seca de grãos (MSGRÃO) do feijoeiro correspondente à produção máxima e $90 \%$ da máxima, doses de Lithothamnium estimadas para promover essas produções e taxa de beneficio (g MSGRÃO/g Lithothamnium aplicado), na dose equivalente a 90\% da MSGRÃO.

\begin{tabular}{|c|c|c|c|c|c|}
\hline Solo & $\begin{array}{l}\text { MSGRÃO } \\
90 \%\end{array}$ & Máximo & $\begin{array}{l}\text { Dose de Lithothamnium } \\
90 \%\end{array}$ & Máxima & Taxa de Benefício \\
\hline \multicolumn{5}{|c|}{ 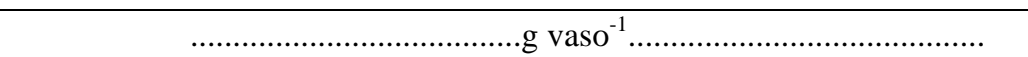 } & g MSGRÃO/g Lithothamnium \\
\hline NQ & 5,58 & 6,20 & 1,53 & 5,06 & 3,64 \\
\hline LVd & 12,90 & 14,33 & 2,74 & 17,04 & 4,71 \\
\hline
\end{tabular}


Pelo Quadro 7 pode-se comparar os tratamentos adicionais com a aplicação de Lithothamnium para $\mathrm{V}$ a $70 \%$. O tratamento calcário dolomítico não foi significativo a 5\% pelo teste $\mathrm{t}$. A adição de $\mathrm{Mg}$ ao Lithothamnium, T9, apesar de fornecer relações $\mathrm{Ca}: \mathrm{Mg}$ no solo similares às do calcário, não surtiu o mesmo efeito desse tratamento (Quadro 7).

A variabilidade dos resultados de MSGRÃO (Quadro 7) é um complicador para a discussão de causas e efeitos dos tratamentos. Por exemplo, Lithothamnium + Mg apresentou a maior produção de MSGRÃO no solo AVd e a menor no solo LVd. Já o tratamento T7, sem micronutrientes na adubação, inviabilizou a produção de grãos no solo NQ e provocou apenas ligeiros acréscimo e decréscimo de produção nos outros dois solos. Esse tratamento, T7, Lithothamnium sem micronutrientes e aumento da V\% e do $\mathrm{pH}$ (Quadro 4), provavelmente reduziu a disponibilidade dos micronutrientes, pois o produto não foi capaz de suplementar esses nutrientes nesse tipo de solo. Deve-se ressaltar que nesse solo (NQ) o seu uso não dispensaria, porém, a aplicação de micronutrientes para a cultura do feijoeiro.

Com o incremento das doses de Lithothamnium, ocorreu aumento linear no teor de $\mathrm{Ca}$ e $\mathrm{Mg}$ nas folhas do feijoeiro, $(\mathrm{P}<0,05)$. Isso seria conseqüência do aumento nos teores de $\mathrm{Ca}$ e $\mathrm{Mg}$ trocável no solo, ocasionado pelo Lithothamnium, possibilitando o aumento de absorção pelo sistema radicular da cultura. As folhas do feijoeiro apresentaram teores de $\mathrm{Ca}$ mais elevados no tratamento T5, que representa o dobro da dose de Lithothamnium para se atingir um $\mathrm{V}$ a $70 \%$, à exceção no solo $\mathrm{AVd}$, em que o T4 foi maior, porém, não diferindo estatisticamente do T5 (Quadro 8).

Entretanto, no T6, calcário dolomítico, os teores de Ca foram menores que no T4 (Lithothamnium $\mathrm{V}$ a $70 \%$ ), porém, não diferiram estatisticamente (Quadro 8). Maiores teores de $\mathrm{Mg}$ nas folhas estiveram associados ao T6, calcário dolomítico, e adicional T9, Lithothamnium mais $\mathrm{Mg}$, nos três solos. De maneira oposta, os menores teores ocorreram no tratamento T1, com ausência de Lithothamnium. O aumento nos teores de $\mathrm{Mg}$ acompanha as doses de Lithothamnium. Ressaltase que, no solo LVd, o adicional T9 apresentou a menor relação $\mathrm{Ca}: \mathrm{Mg}$, evidenciada pelo aumento no teor de $\mathrm{Mg}$, sem, contudo, afetar o teor de Ca. O tratamento T8 (redução em $20 \%$ de NPK), quando comparado ao T4, não diferiu estatisticamente para esses nutrientes.

QUADRO 7 - Produção de matéria seca de grãos (MSGRÃO) do feijoeiro nos tratamentos com Lithothamnium para elevação da $\mathrm{V}$ a $70 \%$ e adicionais.

\begin{tabular}{|c|c|c|c|}
\hline \multirow{2}{*}{ Descrição } & \multicolumn{3}{|c|}{ MSGRÃO } \\
\hline & NQ & LVd & AVd \\
\hline & \multicolumn{3}{|c|}{ 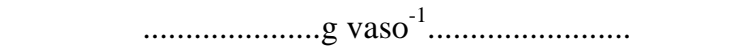 } \\
\hline Lithothamnium- $V_{70}$ & 6,4 & 13,3 & 18,2 \\
\hline Calcário dolomítico $^{1} \mathbf{V}_{70}$ & 7,7 & 14,9 & 25,9 \\
\hline teste $\mathrm{t}$ & 0,29 & $\mathbf{0 , 1 2}$ & $0,06^{\circ}$ \\
\hline Lithothamnium $\mathrm{V}_{70}$ & 6,4 & 13,3 & 18,2 \\
\hline Lithothamnium - micros & 0,0 & 11,5 & 20,3 \\
\hline teste $\mathrm{t}$ & $0,00^{* *}$ & $0,03 *$ & 0,31 \\
\hline Lithothamnium $V_{70}$ & 6,4 & 13,3 & 18,2 \\
\hline Lithothamnium - 20\% NPK & 9,7 & 10,4 & 15,6 \\
\hline teste $t$ & $\mathbf{0 , 0 4 *}$ & $\mathbf{0 , 0 0 * *}$ & 0,29 \\
\hline Lithothamnium $\mathrm{V}_{70}$ & 6,4 & 13,3 & 18,2 \\
\hline Lithothamnium $+\mathrm{Mg}$ & 10,5 & 8,0 & 20,3 \\
\hline teste $\mathrm{t}$ & $0,06^{\circ}$ & $0,00 * *$ & 0,31 \\
\hline
\end{tabular}

${ }^{1}$ Dose de calcário dolomítico $(\mathrm{PN}=102,9 \%){ }^{\circ}, *{ }^{*} *$ significativo a $10 \%, 5 \%$ e $1 \%$, teste $t$. 
QUADRO 8 - Teores foliares de nutrientes no feijoeiro por ocasião do florescimento.

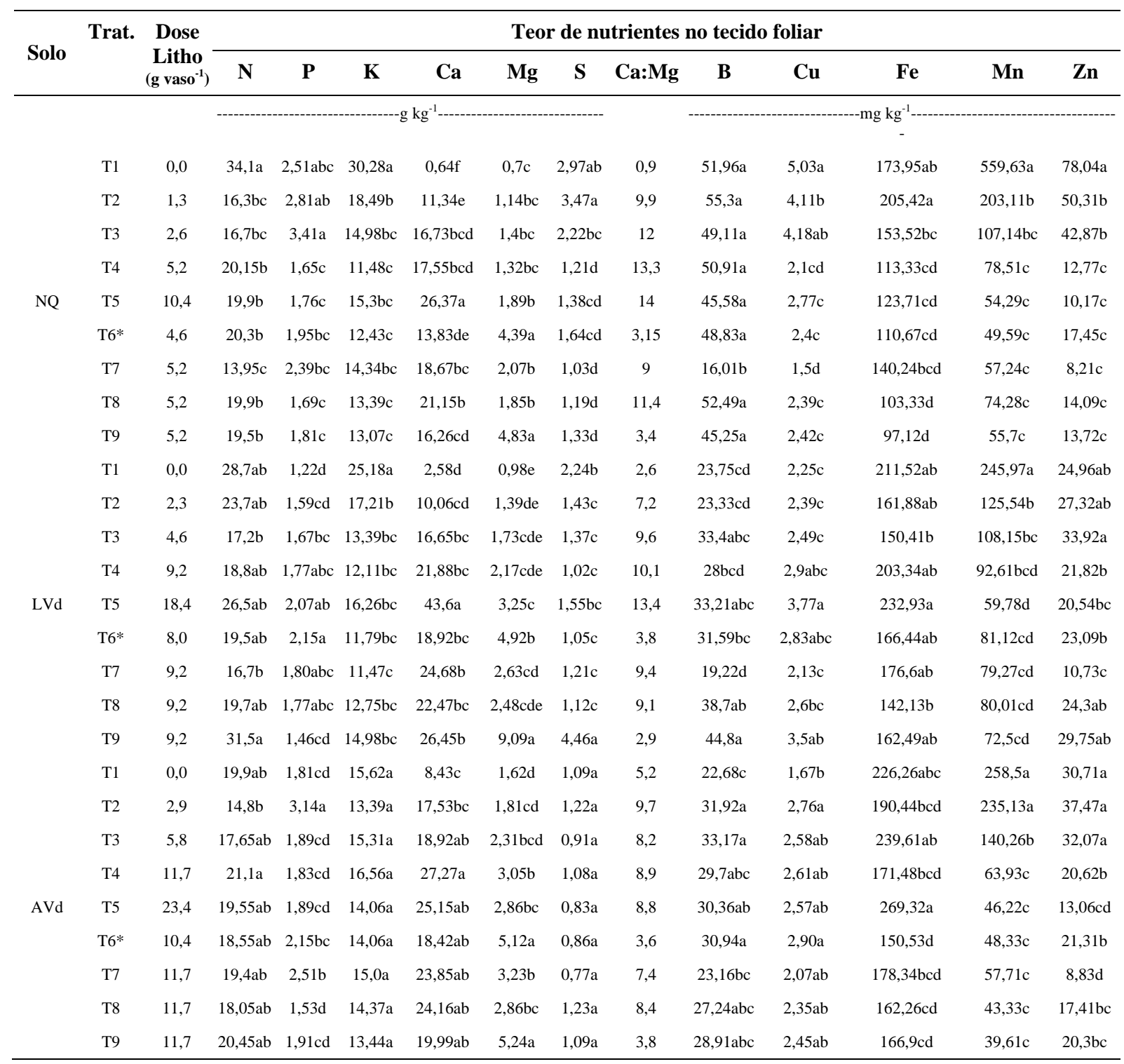

${ }^{1}$ Médias seguidas pela mesma letra na coluna, dentro de cada solo, não diferem entre si (Teste de Tukey $5 \%$ ). * Dose de calcário dolomítico para V a $70 \%$.

Na ausência de Lithothamnium, T1, os teores foliares de $\mathrm{Ca}$ e $\mathrm{Mg}$ encontram-se abaixo do adequado, segundo Malavolta et al. (1997), e tendem a tornar-se adequados com a aplicação do corretivo de origem marinha.

No solo NQ, os teores de Ca e Mg mostraram incrementos de $97,6 \%$ e $63 \%$, entre a testemunha (dose 0 ) e a dose mais elevada $\mathrm{T} 5$, respectivamente. Tais incrementos foram da ordem de $94 \%$ e $69,8 \%$ no solo LVd e de $66,5 \%$ e $43,4 \%$ no solo AVd, para os mesmos nutrientes. No Quadro 9, encontram-se as equações de regressão ajustadas para os teores foliares de $\mathrm{Ca}$ e $\mathrm{Mg}$ do feijoeiro, em função das doses de Lithothamnium e os níveis críticos calculados. 
QUADRO 9 - Equações de regressão ajustadas para teores foliares de $\mathrm{Ca}$ (Y em g kg $\left.{ }^{-1}\right)$ e $\mathrm{Mg}\left(\mathrm{Z} \mathrm{em} \mathrm{g} \mathrm{kg}^{-1}\right)$ na época do florescimento, como variável dependente das doses do Lithothamnium aplicadas (X em $g$ vaso $\left.{ }^{-1}\right)$ e níveis críticos foliares de $\mathrm{Ca}$ e $\mathrm{Mg}$ na dose para produção máxima econômica do feijoeiro.

\begin{tabular}{|c|c|c|c|}
\hline Solo & Equação & $\mathbf{R}^{2}$ & $\begin{array}{l}\text { Níveis críticos } \\
\qquad\left(\mathrm{g} \mathrm{kg}^{-1}\right)\end{array}$ \\
\hline \multirow{2}{*}{ NQ } & $\mathrm{Ca} Y=3,3625+4,5821 \mathrm{X}-0,2335 \mathrm{X}^{2}$ & $0,90 * *$ & 8,4 \\
\hline & $\operatorname{Mg} Y=0,8373+0,1608 X-0,0061 X^{2}$ & $0,86^{* *}$ & 1,0 \\
\hline \multirow{2}{*}{$\mathrm{LVd}$} & $\mathrm{Ca} Y=4,0786+2,226 \mathrm{X}-0,0054 \mathrm{X}^{2}$ & $0,98 * *$ & 7,4 \\
\hline & $\mathrm{Ca} Y=1,0277+0,1427 \mathrm{X}-0,0012 \mathrm{X}^{2}$ & $0,99 * *$ & 1,2 \\
\hline \multirow{2}{*}{$\mathrm{AVd}$} & $\mathrm{Ca} Y=9,2067+2,3609 \mathrm{X}-0,0718 \mathrm{X}^{2}$ & $0,96 * *$ & 14,3 \\
\hline & $\operatorname{Mg} Y=1,4856+0,1861 X-0,0054 X^{2}$ & $0,95 * *$ & 1,9 \\
\hline
\end{tabular}

** significativo a $1 \%$ (teste $\mathrm{t})$.

Os valores de nível critico foliar (Quadro 9) para o $\mathrm{Ca}$, em função de doses de Lithothamnium, variaram de 7,4 a $14,3 \mathrm{~g} \mathrm{~kg}^{-1}$, nos três solos. Esses valores que foram determinados por uma equação quadrática encontram-se abaixo da faixa preconizada por Malavolta et al. (1997). Para o Mg, também em função das doses, os valores variaram de 1,0 a $1,9 \mathrm{~g} \mathrm{~kg}^{-1}$, portanto, dentro da faixa adequada, considerada por esse autor.

O aumento da dose de Lithothamnium ocasionou redução nos teores foliares de $\mathrm{Mn}$ e $\mathrm{Zn}$, principalmente na maior dose, T5 (Quadro 8). Esse fato está ligado à reação do corretivo, provocando aumentos nos valores de $\mathrm{pH}$, originando formas insolúveis desses nutrientes em detrimento das formas solúveis absorvidas pela plantas $\left(\mathrm{Mn}^{+2}\right.$ e $\left.\mathrm{Zn}^{+2}\right)$. Esse aspecto é freqüentemente associado às perdas de produtividade devido à supercalagem (Raij, 1991) e, possivelmente, explique os resultados obtidos com o feijoeiro.

\section{Absorção e acúmulo de macronutrientes na matéria seca da parte aérea}

Os tratamentos provocaram alterações significativas na absorção de alguns macro e micronutrientes (Quadro 10). As maiores absorções de Ca estão associadas ao T5 e, para o Mg, aos tratamentos T6 e T9, nos três solos. Já as menores absorções de Ca e Mg ocorreram no nível zero de Lithothamnium (T1), nos três solos. Isso sugere que uma maior absorção de tais nutrientes é atingida com o suprimento de $\mathrm{Ca}$ e $\mathrm{Mg}$, fornecido pela aplicação de Lithothamnium.

A baixa produção de matéria seca na ausência de Lithothamnium (T1) contribuiu para um menor acúmulo dos nutrientes $\mathrm{Ca}$ e Mg na planta. Por outro lado, a dose de Lithothamnium para se atingir um V a 70\% (T4) não diferiu estatisticamente $(\mathrm{P}<0,05)$ da dose equivalente de calcário dolomítico (T6), para um V a 70\%.

A relação $\mathrm{Ca}: \mathrm{Mg}$ na matéria seca da parte aérea apresentou grande variação entre os tratamentos na presença do Lithothamnium. Esses valores variaram desde 7,5 até $15: 1$, sendo menores nos tratamentos com calcário dolomítico (T6) e adição de magnésio ao Lithothamnium (T9), com valores de 3 até 3,9:1 (Quadro 10).

A relação entre $\mathrm{Ca}$ e $\mathrm{Mg}$ para o feijoeiro parece não ter tamanha importância, pois, nos tratamentos T4 e T6 (Quadro 10), a relação foi de 12 e 3,5:1 no solo NQ; de 10,6 e 3,9:1 no solo LVd e de 8,4 e 3,7:1 no solo $\mathrm{AVd}$, característico de planta calcícola.

Peñalosa et al. (1995) constataram uma relação direta em plantas de feijão entre absorção de Ca e K com a relação $\mathrm{Ca}: \mathrm{K}$ da solução. Resultados obtidos por Fernandes (2000), em que a equivalência na solução entre $\mathrm{Ca}$ e $\mathrm{K}$ foi de $1: 2 \mathrm{mmol} \mathrm{L}^{-1}$, proporcionaram maiores acúmulos de $\mathrm{K}$, Ca e $\mathrm{S}$ na planta. Os resultados obtidos não corroboram com Fernandes (2000), pois com o aumento na absorção de $\mathrm{Ca}$, os acúmulos de K e S praticamente não se alteraram (Quadro 10). 
QUADRO 10 - Acúmulo de macronutriente na matéria seca da parte aérea do feijoeiro nos diferentes solos e doses do Lithothamnium.

\begin{tabular}{|c|c|c|c|c|c|c|c|c|c|}
\hline Solo & Trat. & Dose & $\mathbf{N}$ & $\mathbf{P}$ & $\mathbf{K}$ & $\mathbf{C a}$ & Mg & $\mathbf{S}$ & Ca:Mg \\
\hline & \multicolumn{9}{|c|}{ 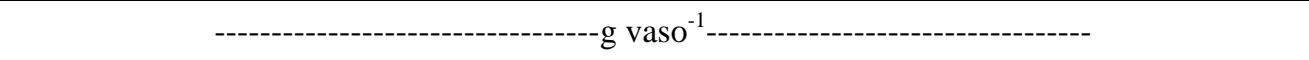 } \\
\hline \multirow[t]{9}{*}{ NQ } & $\mathrm{T} 1$ & 0,0 & $0,30 \mathrm{a}$ & $0,023 \mathrm{c}$ & $0,27 \mathrm{a}$ & $0,06 \mathrm{c}$ & $0,01 \mathrm{f}$ & $0,02 \mathrm{c}$ & 6 \\
\hline & $\mathrm{T} 2$ & 1,3 & $0,29 \mathrm{a}$ & $0,050 \mathrm{abc}$ & $0,33 \mathrm{a}$ & $0,20 \mathrm{~b}$ & $0,02 \mathrm{ef}$ & $0,06 \mathrm{a}$ & 10 \\
\hline & $\mathrm{T} 3$ & 2,6 & $0,31 \mathrm{a}$ & $0,066 \mathrm{a}$ & $0,28 \mathrm{a}$ & $0,31 \mathrm{ab}$ & $0,03 \mathrm{de}$ & $0,04 \mathrm{~b}$ & 10 \\
\hline & $\mathrm{T} 4$ & 5,2 & $0,41 \mathrm{a}$ & $0,034 \mathrm{bc}$ & $0,23 \mathrm{a}$ & $0,36 \mathrm{ab}$ & $0,03 \mathrm{de}$ & $0,02 \mathrm{c}$ & 12 \\
\hline & $\mathrm{T} 5$ & 10,4 & $0,31 \mathrm{a}$ & $0,028 b c$ & $0,23 \mathrm{a}$ & $0,45 \mathrm{a}$ & $0,03 \mathrm{de}$ & $0,02 \mathrm{c}$ & 15 \\
\hline & $\mathrm{T} 6 *$ & 4,6 & $0,41 \mathrm{a}$ & 0041abc & $0,25 \mathrm{a}$ & $0,28 \mathrm{ab}$ & $0,08 \mathrm{~b}$ & $0,03 b c$ & 3,5 \\
\hline & $\mathrm{T} 7$ & 5,2 & $0,33 a$ & $0,058 \mathrm{ab}$ & $0,35 \mathrm{a}$ & $0,45 \mathrm{a}$ & $0,05 \mathrm{c}$ & $0,02 \mathrm{c}$ & 9 \\
\hline & $\mathrm{T} 8$ & 5,2 & $0,44 \mathrm{a}$ & $0,037 \mathrm{abc}$ & $0,29 \mathrm{a}$ & $0,46 a$ & $0,04 \mathrm{~cd}$ & $0,02 \mathrm{c}$ & 11,5 \\
\hline & T9 & 5,2 & $0,48 \mathrm{a}$ & $0,044 \mathrm{abc}$ & $0,32 \mathrm{a}$ & $0,40 \mathrm{a}$ & $0,12 \mathrm{a}$ & $0,03 b c$ & 3,3 \\
\hline \multirow[t]{9}{*}{$\mathrm{LVd}$} & $\mathrm{T} 1$ & 0,0 & $0,47 a$ & $0,02 \mathrm{~d}$ & $0,41 \mathrm{a}$ & $0,04 \mathrm{f}$ & $0,01 \mathrm{e}$ & $0,04 \mathrm{~b}$ & 4 \\
\hline & $\mathrm{T} 2$ & 2,3 & $0,55 \mathrm{a}$ & $0,04 \mathrm{bcd}$ & $0,41 \mathrm{a}$ & $0,24 \mathrm{e}$ & $0,03 \mathrm{de}$ & $0,03 b$ & 8 \\
\hline & $\mathrm{T} 3$ & 4,6 & $0,47 \mathrm{a}$ & $0,05 \mathrm{abc}$ & $0,36 a b$ & $0,45 \mathrm{~d}$ & $0,05 \mathrm{~cd}$ & $0,04 \mathrm{~b}$ & 9 \\
\hline & $\mathrm{T} 4$ & 9,2 & $0,55 \mathrm{a}$ & $0,05 a b c$ & $0,35 \mathrm{ab}$ & $0,64 b c$ & $0,06 \mathrm{bc}$ & $0,03 b$ & 10,6 \\
\hline & $\mathrm{T} 5$ & 18,4 & $0,60 \mathrm{a}$ & $0,05 \mathrm{abc}$ & $0,36 a b$ & $0,97 \mathrm{a}$ & $0,07 \mathrm{~b}$ & $0,04 \mathrm{~b}$ & 13,8 \\
\hline & $\mathrm{T} 6 *$ & 8,0 & $0,60 \mathrm{a}$ & $0,07 \mathrm{a}$ & $0,36 \mathrm{ab}$ & $0,58 \mathrm{bcd}$ & $0,15 \mathrm{a}$ & $0,03 b$ & 3,9 \\
\hline & $\mathrm{T} 7$ & 9,2 & $0,44 a$ & $0,05 \mathrm{abc}$ & $0,31 \mathrm{ab}$ & $0,66 b$ & $0,07 \mathrm{bc}$ & $0,03 b$ & 9,4 \\
\hline & $\mathrm{T} 8$ & 9,2 & $0,51 \mathrm{a}$ & $0,05 a b c$ & $0,33 \mathrm{ab}$ & $0,59 \mathrm{bcd}$ & $0,06 \mathrm{bc}$ & $0,03 b$ & 9,8 \\
\hline & T9 & 9,2 & $0,57 \mathrm{a}$ & $0,03 \mathrm{~cd}$ & $0,27 \mathrm{~b}$ & $0,48 \mathrm{~cd}$ & $0,16 \mathrm{a}$ & $0,08 \mathrm{a}$ & 3 \\
\hline \multirow[t]{9}{*}{$\mathrm{AVd}$} & $\mathrm{T} 1$ & 0,0 & $0,34 \mathrm{~b}$ & $0,03 d$ & $0,27 \mathrm{~b}$ & $0,15 \mathrm{c}$ & $0,03 \mathrm{~d}$ & $0,02 \mathrm{a}$ & 5 \\
\hline & $\mathrm{T} 2$ & 2,9 & $0,35 \mathrm{ab}$ & $0,07 \mathrm{ab}$ & $0,32 \mathrm{ab}$ & $0,42 b c$ & $0,04 \mathrm{~cd}$ & $0,03 \mathrm{a}$ & 10,5 \\
\hline & $\mathrm{T} 3$ & 5,8 & $0,50 \mathrm{ab}$ & $0,06 \mathrm{abcd}$ & $0,44 \mathrm{ab}$ & $0,55 \mathrm{ab}$ & $0,06 \mathrm{bcd}$ & $0,02 \mathrm{a}$ & 9,2 \\
\hline & $\mathrm{T} 4$ & 11,7 & $0,49 \mathrm{ab}$ & $0,04 \mathrm{bcd}$ & $0,37 \mathrm{ab}$ & $0,59 \mathrm{ab}$ & $0,07 \mathrm{bcd}$ & $0,02 \mathrm{a}$ & 8,4 \\
\hline & $\mathrm{T} 5$ & 23,4 & $0,58 \mathrm{ab}$ & $0,06 a b c d$ & $0,41 \mathrm{ab}$ & $0,75 \mathrm{a}$ & $0,08 \mathrm{bc}$ & $0,03 \mathrm{a}$ & 9,4 \\
\hline & $\mathrm{T} 6 *$ & 10,4 & $0,57 \mathrm{ab}$ & $0,07 \mathrm{ab}$ & $0,43 a b$ & $0,60 \mathrm{ab}$ & $0,16 \mathrm{a}$ & $0,03 \mathrm{a}$ & 3,7 \\
\hline & $\mathrm{T} 7$ & 11,7 & $0,61 \mathrm{a}$ & $0,08 \mathrm{a}$ & $0,47 \mathrm{a}$ & $0,75 \mathrm{a}$ & $0,10 \mathrm{~b}$ & $0,02 \mathrm{a}$ & 7,5 \\
\hline & $\mathrm{T} 8$ & 11,7 & $0,44 a b$ & $0,04 \mathrm{~cd}$ & $0,35 \mathrm{ab}$ & $0,59 \mathrm{ab}$ & $0,07 \mathrm{bcd}$ & $0,03 \mathrm{a}$ & 8,4 \\
\hline & T9 & 11,7 & $0,61 \mathrm{a}$ & $0,06 \mathrm{abcd}$ & $0,40 \mathrm{ab}$ & $0,59 \mathrm{ab}$ & $0,16 \mathrm{a}$ & $0,03 a$ & 3,7 \\
\hline
\end{tabular}

Médias seguidas pela mesma letra minúscula na coluna, dentro de cada solo, não diferem entre si (teste de Tukey 5\%), * calcário dolomítico para V a 70\% (PN=102,9\%), 
De maneira geral, os acúmulos dos macronutrientes na matéria seca da parte aérea das plantas do feijoeiro, à exceção do $\mathrm{Ca}$ e $\mathrm{Mg}$, apresentaram pequena ou nenhuma variação com as doses do Lithothamnium (Quadro 10). Isso provavelmente se deve ao efeito de diluição de nutrientes nos tratamentos que favoreceram maior produção de matéria seca (Jarrel \& Beverly, 1981).

\section{CONCLUSÕES}

Nas condições em que foi realizado esse experimento, conclui-se que:

O Lithothamnium promoveu efeito na correção da acidez do solo;

b) Para atingir as melhores respostas, o feijoeiro não necessitou de condições elevadas na saturação por bases, dado por modelo raiz quadrada;

c) Houve efeito de doses de Lithothamnium no crescimento e produção do feijoeiro;

d) A melhor dose para se atingir $90 \%$ da produção máxima de grãos no solo NQ foi de $0,61 \mathrm{t} \mathrm{ha}^{-1} \mathrm{e}$, no solo $\mathrm{LVd}$, de 1,09 $\mathrm{t} \mathrm{ha}^{-1}$, bem abaixo das doses para $\mathrm{V}$ a $70 \%$.

\section{REFERÊNCIAS BIBLIOGRÁFICAS}

BANZATO, D. A.; KRONKA, S. N. Experimentação agrícola. 2. ed. Jaboticabal: FUNEP, 1989. 247 p.

BERGER, K. C.; TRUOG, E. Boron determination in soils and plants. Industrial and Engineering Chemistry, Washington, v. 11, p. 540-545, 1939.

BORNMAN, J. J.; BORNMAN, L.; BARNARD, O. The effects of calcium carbonate and calcium hydroxide on plant growth during overliming. Soil Science, Baltimore, v. 163, n. 6, p. 498-507, June 1998.

BRASIL. Ministério da Agricultura. Secretaria Nacional de defesa Agropecuária. Análise de corretivos, fertilizantes e inoculantes: métodos oficiais. Brasília: LANARV/SNAD, 1983. 104 p.

FEITOSA, C. T. Adubação NP para o feijoeiro (Phaseolus vulgaris L.) na presença e na ausência de calcário. Revista Brasileira de Ciência do Solo, Campinas, v. 4, n. 3, p. 156-159, 1980.

FREIRE, J. C.; RIBEIRO, M. A. V.; BAHIA, V. G.; LOPES, A. S.; AQUINO, L. E. Resposta do milho cultivado em casa-de-vegetação a níveis de água em solos da região de Lavras-MG. Revista Brasileira de Ciência do Solo, Campinas, v. 4, n. 5, p. 5-8, jan./abr. 1980.
FERNANDES, A. R. Nutrição mineral e crescimento de mudas de pupunheira (Bactris gasipaes H.B.K.), em solução nutritiva, em função do balanço de nutrientes e níveis de salinidade. 2000. 145 p. Tese (Doutorado em Solos e Nutrição de plantas) - Universidade Federal de Lavras, Lavras.

JARREL, W. M.; BEVERLY, R. B. The dilution effect in plant nutrition studies. Advances in Agronomy, New York, v. 34, p. 197-224, 1981.

KEMPF, M. Perspectivas de exploração econômica dos Fundos de algas calcárias da plataforma continental do Nordeste do Brasil. Recife: Universidade Federal de Pernambuco, 1974. 22 p. (Trabalho oceanográfico, 14).

LE BLEU, P. Contribution à l'étude des algues marines en Bretagne: bilan de leur utilisation em milieu agricole. France: Tours, 1983. 103 p.

LOPEZ-BENITO, M. Estudio de la composición química del Lithothamnium calcareum (Aresch) y su aplicación como corrector de terrenos de cultivo. Inv. Pesq., [S.1.], v. 23, p. 53-70, jun. 1963.

MALAVOLTA, E.; VITTI, G. C.; OLIVEIRA, S. A. Avaliação do estado nutricional das plantas: princípios e aplicações. 2. ed. Piracicaba: Ceres, 1997. $319 \mathrm{p}$.

MIRANDA, L. N. Utilização de calcários marinhos como corretivos de acidez do solo. Revista Brasileira de Ciência do Solo, Campinas, v. 9, n. 1, p. 245-248, jan./mar. 1985.

PEÑAlOSA, J. M.; CÁCERES, M. D.; SARRO, M. J. Nutrition of beans plants in sand culture: influence of calcium/potassium ratio in the nutrient solution. Journal of Plant Nutrition, New York, v. 18, n. 10, p. 2023-2032, 1995.

RAIJ, B. van. Fertilidade do solo e adubação. Piracicaba: CERS/POTAFOS, 1991. 342 p.

RAIJ, B. van; CANTARELLA, H.; QUAGGIO, J. A.; FURLANI, A. M. C. Recomendações de adubação e calagem para o estado de São Paulo. 2. ed. Campinas: IAC, 1996. 285 p. (Boletim Técnico, 100).

ROSOLEM, C. A. Nutrição e adubação do feijoeiro. Piracicaba: POTAFÓS, 1987.93 p. 